\title{
A Search for IceCube Neutrinos from the First 33 Detected Gravitational Wave Events
}

\section{The IceCube Collaboration*}

http://icecube.wisc.edu/collaboration/authors/icrc19_icecube

E-mail: rhussaineicecube.wisc.edu

\begin{abstract}
The discoveries of high-energy astrophysical neutrinos by IceCube in 2013 and of gravitational waves by LIGO in 2015 have enabled a new era of multi-messenger astronomy. Gravitational waves can identify the merging of compact objects such as neutron stars and black holes. These compact mergers, especially neutron star mergers, are potential neutrino sources. We present an analysis searching for neutrinos from gravitational wave sources reported by the LIGO Virgo Collaboration (LVC). We use a dedicated transient likelihood analysis combining IceCube events with source localizations provided by $\mathrm{LVC}$ as spatial priors. We report results for all gravitational wave events from the $\mathrm{O} 1, \mathrm{O} 2$, and $\mathrm{O} 3$ observing runs.
\end{abstract}

Corresponding authors: Raamis Hussain ${ }^{\dagger 1}$, Justin Vandenbroucke ${ }^{1}$, Joshua Wood ${ }^{2}$

${ }^{1}$ University of Wisconsin - Madison

${ }^{2}$ IceCube Collaboration

36th International Cosmic Ray Conference -ICRC2019-

July 24th - August 1st, 2019

Madison, WI, U.S.A.

\footnotetext{
*For collaboration list, see PoS(ICRC2019) 1177.

${ }^{\dagger}$ Speaker.
} 


\section{Introduction}

With the release of the first Gravitational Wave Transients Catalog [1] and the start of the $\mathrm{O} 3$ observing run of the LIGO-Virgo Collaboration (LVC), there have been 33 detected compact binary mergers as of July 28th, 2019. Of these 33 detections, 28 have been classified as binary black hole mergers (BBH), 3 are thought to be binary neutron star mergers (BNS), and 2 events may be of terrestrial origin [2].

Although several multi-messenger campaigns to find electromagnetic counterparts to gravitational waves (GWs) have been conducted, only one successful multi-messenger detection has been made [3]. The observation of GW170817, and the associated short gamma-ray burst GRB170817A, provided a wealth of knowledge about the physical processes and dynamics of the astrophysical system $[4,5]$.

Neutrino follow up searches of gravitational wave events can provide information complementary to electromagnetic counterparts. For example, neutrinos can provide information about particle acceleration mechanisms [6], jet dynamics [7], and the environment near the source [8]. In addition to providing insight in to the physics of these sources, detection of neutrinos from GW sources can greatly improve the localization compared to detection of GWs alone. Searches for joint GW and high-energy neutrino events have produced no significant detections to date $[4,9,10]$.

IceCube is a cubic kilometer neutrino observatory located at the geographic South Pole. It has a duty cycle close to $100 \%$ and is able to observe the full sky at all times [11]. Rapid analysis of neutrino data from IceCube presents a unique opportunity to quickly identify joint sources of GWs and neutrinos and to report these findings to electromagnetic telescopes for further study.

In these proceedings, we present a comprehensive neutrino follow up to every detected GW event from the LVC O1, O2 and partially completed $\mathrm{O} 3$ observing run. The unbinned maximum likelihood used for the search is described in Section 2. The data used in this analysis are described in Section 3. The results for each follow up are summarized in Section 4. We conclude with a discussion of the results and future work in Section 5.

\section{Method}

We perform an unbinned maximum likelihood analysis which uses the LVC skymap as a spatial prior. The likelihood we use is

$$
L=\frac{e^{-\left(n_{\mathrm{s}}+n_{\mathrm{b}}\right)}\left(n_{\mathrm{s}}+n_{\mathrm{b}}\right)^{N}}{N !} \prod_{i=1}^{N} \frac{n_{\mathrm{s}} S_{i}\left(x_{i}, E_{i} ; \gamma\right)+n_{\mathrm{b}} B_{i}\left(x_{i}, E_{i}\right)}{n_{\mathrm{s}}+n_{\mathrm{b}}}
$$

where $N$ is the total number of observed neutrino events, $n_{\mathrm{b}}$ is the expected number of background events, $n_{\mathrm{s}}$ is the number of signal events, and $\gamma$ is the spectral index of the source. For an event with a given energy $E_{i}$ and direction $x_{i}$, we form a PDF of signal correlation $S_{i}\left(x_{i}, E_{i} ; \gamma\right)$ and a PDF of background correlation $B_{i}\left(x_{i}, E_{i}\right)$. Both the signal and background PDFs consist of a spatial and energy component

$$
S_{i}=S_{\text {space }}\left(x_{i}, \sigma_{i} \mid x_{\mathrm{s}}\right) \cdot S_{\text {energy }}\left(x_{i}, E_{i} \mid \gamma\right) ; \quad B_{i}=B_{\text {space }}\left(x_{i}\right) \cdot B_{\text {energy }}\left(x_{i}, E_{i}\right)
$$


We divide the sky into equal-area bins using HEALPix [12]. The pixels are roughly $0.01 \mathrm{deg}^{2}$. In the equations above, the location of the pixel being tested is $x_{\mathrm{s}}$, while $x_{i}$ and $\sigma_{i}$ are the reconstructed direction and estimated angular uncertainty of each neutrino event.

The test statistic is the log of the likelihood ratio

$$
\mathrm{TS}=2 \ln \left(\frac{L\left(\hat{n}_{\mathrm{s}}, \hat{\gamma}\right)}{L\left(n_{\mathrm{s}}=0\right)}\right) ; \quad w=\frac{P_{\mathrm{GW}}\left(x_{\mathrm{s}}\right)}{\Omega_{\text {pixel }}}
$$

where $\hat{n}_{\mathrm{s}}$ and $\hat{\gamma}$ are the free parameters in the maximum likelihood fit.

We incorporate the GW spatial prior by defining a weight, shown above, for every pixel in the skymap. This weight represents the probability density of the GW source being at a given position in the sky. The weight is then normalized and multiplied by the signal likelihood which results in a modified test statistic

$$
\Lambda=2 \ln \left(\frac{L\left(\hat{n}_{\mathrm{s}}, \hat{\gamma}\right) \cdot w}{L\left(n_{\mathrm{s}}=0\right)}\right)=\mathrm{TS}+2 \ln (w)=2\left(-n_{\mathrm{s}}+\sum_{i=1}^{N} \ln \left[1+\frac{n_{\mathrm{s}} S_{i}}{n_{\mathrm{b}} B_{i}}\right]\right)+2 \ln (w)
$$

To test for a $\mathrm{GW}+$ neutrino coincidence, we consider a $\pm 500 \mathrm{~s}$ time window centered on the $\mathrm{GW}$ event time. This is a conservative time window derived by considering a range of prompt neutrino emission mechanisms from gamma-ray bursts [13]. We perform a scan over the full sky and maximize the likelihood ratio with respect to $n_{\mathrm{s}}$ and $\gamma$ at every pixel. The largest test statistic in the sky is considered the best fit position for that scan.

To calculate the significance of a given observation, we compute a p-value by comparing our observed $\Lambda$ to a background distribution which is built from 30,000 trials using randomized neutrinos and a fixed gravitational wave skymap for each trial. Neutrinos are randomized by scrambling their arrival time and recomputing their direction based on their new randomly assigned time. All trials use the same GW skymap. An example background distribution is shown in Figure 1. This pvalue quantifies the significance of the observed neutrinos being associated with a point-like source located within the given GW skymap.

To compute our sensitivity for each GW, we perform signal injection trials and compute the flux required such that $90 \%$ of trials return an observed $\Lambda$ greater than the median $\Lambda$ of the background distribution. Signal neutrinos are selected from Monte Carlo and injected according to an $E^{-2}$ power law spectrum. An example of this procedure is shown in Figure 2.

\section{Data Sample}

Neutrino data used in this analysis come from the IceCube Gamma-ray Follow Up (GFU) dataset, which is a sample of through-going muon tracks used for realtime analyses [11]. The sample has a $6.7 \mathrm{mHz}$ all-sky event rate with the dominant background in the Southern and Northern Hemispheres coming from atmospheric muons and atmospheric neutrinos, respectively. The sample has a median angular resolution of $\lesssim 1 \mathrm{deg}$ for neutrino energies above $1 \mathrm{TeV}$ [14]. 


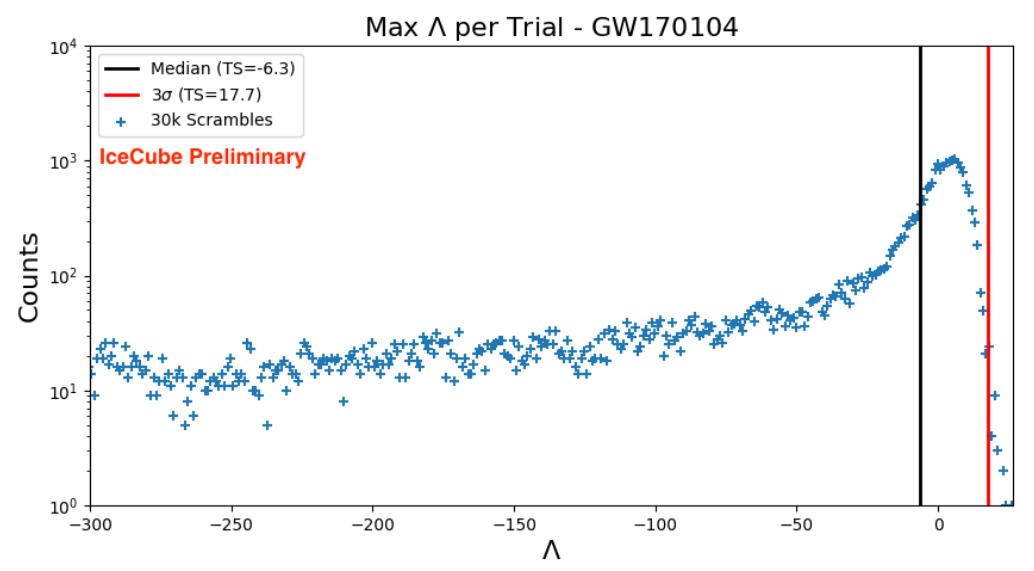

Figure 1: Background TS distribution for GW170104. The distribution is constructed by taking the maximum $\Lambda$ per trial for 30k background-only trial. More than half of the trials return negative $\Lambda$ values since most of the pixels in the sky have a very low GW spatial prior weight, $w$, from the GW PDF and thus are heavily penalized.

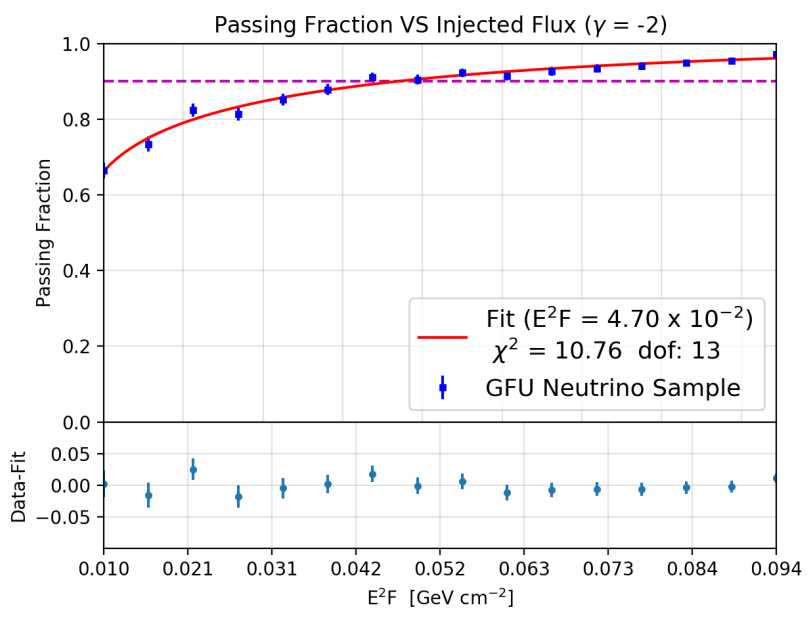

Figure 2: 90\% Sensitivity flux for GW170104, computed by injecting an increasing neutrino flux according to an $E^{-2}$ power law spectrum and calculating the fraction of trials which return an observed $\Lambda$ greater than the median of the background distribution shown in Figure 1 . We use a $\chi^{2}$ cumulative distribution function $(\mathrm{CDF})$ to fit the data and compute the flux which gives a $90 \%$ passing fraction. This is defined as the sensitivity for the particular GW event.

\section{Results}

Table 1 summarizes the final results for each GW event. No significant neutrino correlation was found. We report $90 \%$ C.L. upper limits (U.L.s) on the time-integrated neutrino flux scaled by event energy, $E^{2} F$, from each GW event. We also report $90 \%$ U.L.s on the isotropic equivalent energy emitted in neutrinos within the $\pm 500 \mathrm{~s}$ time window, assuming the median value of the reported luminosity distance to the GW source.

The flux U.L.s are computed using the same procedure as the $90 \%$ sensitivity flux. The dif- 
ference between the U.L. and the sensitivity is that the U.L. uses the observed $\Lambda$ as the threshold rather than the median $\Lambda$ of the background. In cases where the observed $\Lambda$ is less than the median, we use the median $\Lambda$ as our threshold to be conservative.

We also report U.L.s on the isotropic equivalent energy, $E_{\text {iso }}$, emitted in neutrinos over the $1000 \mathrm{~s}$ time window for each GW. This limit is derived using a relation between $E_{\text {iso }}$ and the mean number of expected events at IceCube, taking into account the effective area of the detector and assuming an $\mathrm{E}^{-2}$ powerlaw spectrum. We also marginalize over the $3 \mathrm{D}$ position of the source using skymaps and distance PDFs provided by LVC. $E_{\text {iso }}$ is tuned such that the mean number of expected neutrino events at IceCube is 2.3 , which corresponds to the $90 \%$ C.L. Poisson U.L. given a non-observation of neutrinos at IceCube.

\begin{tabular}{|c|c|c|c|c|c|c|c|c|}
\hline \multicolumn{9}{|c|}{ GW Event List } \\
\hline Event & Type & $\Omega\left(\operatorname{deg}^{2}\right)$ & FAR $\left(\mathrm{yr}^{-1}\right)$ & p-Value & $\begin{array}{l}\text { 90\% U.L. } \\
\left(\mathrm{GeVcm}^{-2}\right)\end{array}$ & $\begin{array}{l}90 \% \text { U.L.min } \\
\left(\mathrm{GeVcm}^{-2}\right)\end{array}$ & $\begin{array}{l}\text { 90\% U.L.max } \\
\left(\mathrm{GeVcm}^{-2}\right)\end{array}$ & $E_{\text {iso }}(\mathrm{ergs})$ \\
\hline GW150914 & $\mathrm{BBH}$ & 180 & $<1.00 \times 10^{-7}$ & 0.62 & 0.432 & 0.0296 & 1.03 & $1.60 \times 10^{52}$ \\
\hline GW151012 & $\mathrm{BBH}$ & 1555 & $7.92 \times 10^{-3}$ & 0.71 & 0.177 & 0.0286 & 0.821 & $8.14 \times 10^{52}$ \\
\hline GW151226 & $\mathrm{BBH}$ & 1033 & $<1.00 \times 10^{-7}$ & 0.79 & 0.205 & 0.0286 & 0.904 & $1.45 \times 10^{52}$ \\
\hline GW170104 & $\mathrm{BBH}$ & 924 & $<1.00 \times 10^{-7}$ & 0.54 & 0.0440 & 0.0286 & 0.667 & $7.11 \times 10^{52}$ \\
\hline GW170608 & $\mathrm{BBH}$ & 396 & $<1.00 \times 10^{-7}$ & 0.61 & 0.0365 & 0.0309 & 0.0821 & $9.40 \times 10^{51}$ \\
\hline GW170729 & $\mathrm{BBH}$ & 1033 & $1.80 \times 10^{-1}$ & 0.21 & 0.620 & 0.0286 & 1.02 & $5.39 \times 10^{53}$ \\
\hline GW170809 & $\mathrm{BBH}$ & 340 & $<1.00 \times 10^{-7}$ & 0.60 & 0.270 & 0.0568 & 0.758 & $8.21 \times 10^{52}$ \\
\hline GW170814 & $\mathrm{BBH}$ & 87 & $<1.00 \times 10^{-7}$ & 1.0 & 0.449 & 0.488 & 0.711 & $2.94 \times 10^{52}$ \\
\hline GW170817 & BNS & 16 & $<1.00 \times 10^{-7}$ & 0.19 & 0.274 & 0.180 & 0.429 & $1.37 \times 10^{50}$ \\
\hline GW170818 & $\mathrm{BBH}$ & 39 & $4.20 \times 10^{-5}$ & 0.52 & 0.0276 & 0.0364 & 0.0431 & $9.04 \times 10^{52}$ \\
\hline GW170823 & $\mathrm{BBH}$ & 1651 & $<1.00 \times 10^{-7}$ & 0.75 & 0.182 & 0.0286 & 0.796 & $2.46 \times 10^{53}$ \\
\hline S190408an & BBH & 387 & $<1.00 \times 10^{-7}$ & 0.13 & 0.0625 & 0.0337 & 0.606 & $1.81 \times 10^{53}$ \\
\hline S190412m & $\mathrm{BBH}$ & 156 & $<1.00 \times 10^{-7}$ & 0.18 & 0.0423 & 0.0286 & 0.048 & $5.39 \times 10^{52}$ \\
\hline S190421ar & $\mathrm{BBH}$ & 1444 & $4.70 \times 10^{-1}$ & 0.79 & 0.652 & 0.0420 & 1.15 & $1.65 \times 10^{53}$ \\
\hline S190425z & BNS & 7461 & $1.43 \times 10^{-5}$ & 0.87 & 0.383 & 0.0286 & 1.06 & $1.90 \times 10^{51}$ \\
\hline S190426c & BNS & 1131 & $6.14 \times 10^{-1}$ & 0.12 & 0.0685 & 0.0286 & 0.583 & $1.10 \times 10^{52}$ \\
\hline S190503bf & $\mathrm{BBH}$ & 448 & $5.16 \times 10^{-2}$ & 0.49 & 0.581 & 0.227 & 0.821 & $1.43 \times 10^{52}$ \\
\hline S190510g & Ter & 1166 & $2.79 \times 10^{-1}$ & 0.86 & 0.401 & 0.0286 & 0.610 & $2.76 \times 10^{51}$ \\
\hline S190512at & $\mathrm{BBH}$ & 252 & $6.00 \times 10^{-2}$ & 0.84 & 0.341 & 0.0286 & 0.568 & $1.51 \times 10^{53}$ \\
\hline S190513bm & $\mathrm{BBH}$ & 691 & $1.18 \times 10^{-5}$ & 1.0 & 0.187 & 0.0286 & 0.505 & $3.16 \times 10^{53}$ \\
\hline S190517h & $\mathrm{BBH}$ & 939 & $7.49 \times 10^{-2}$ & 0.21 & 0.613 & 0.0286 & 1.06 & $5.78 \times 10^{53}$ \\
\hline S190519bj & $\mathrm{BBH}$ & 967 & $1.80 \times 10^{-1}$ & 0.45 & 0.108 & 0.0286 & 0.639 & $8.15 \times 10^{53}$ \\
\hline S190521g & BBH & 765 & $1.20 \times 10^{-1}$ & 0.61 & 0.538 & 0.0391 & 0.966 & $1.14 \times 10^{54}$ \\
\hline S190521r & $\mathrm{BBH}$ & 488 & $1.00 \times 10^{-2}$ & 0.095 & 0.0654 & 0.0286 & 0.456 & $1.07 \times 10^{53}$ \\
\hline S190602aq & $\mathrm{BBH}$ & 1172 & $<1.00 \times 10^{-7}$ & 0.15 & 0.344 & 0.0286 & 0.732 & $4.84 \times 10^{52}$ \\
\hline S190630ag & $\mathrm{BBH}$ & 1483 & $4.53 \times 10^{-6}$ & 0.63 & 0.307 & 0.0286 & 0.977 & $6.73 \times 10^{52}$ \\
\hline S190701ah & $\mathrm{BBH}$ & 67 & $6.04 \times 10^{-1}$ & 1.0 & 0.0530 & 0.0286 & 0.176 & $2.81 \times 10^{53}$ \\
\hline S190706ai & $\mathrm{BBH}$ & 1100 & $6.00 \times 10^{-2}$ & 1.0 & 0.199 & 0.0350 & 0.881 & $2.09 \times 10^{54}$ \\
\hline S190707q & $\mathrm{BBH}$ & 1375 & $1.66 \times 10^{-4}$ & 0.55 & 0.334 & 0.0286 & 0.763 & $4.99 \times 10^{52}$ \\
\hline S190718y & Ter & 7246 & 1.15 & 0.67 & 0.135 & 0.0286 & 1.15 & $1.42 \times 10^{51}$ \\
\hline S190720a & $\mathrm{BBH}$ & 1559 & 0.120 & 0.96 & 0.358 & 0.0286 & 1.08 & $6.29 \times 10^{52}$ \\
\hline S190727h & $\mathrm{BBH}$ & 841 & $4.34 \times 10^{-3}$ & 0.95 & 0.592 & 0.0350 & 0.983 & $6.98 \times 10^{53}$ \\
\hline S190728q & $\mathrm{BBH}$ & 104 & $<1.00 \times 10^{-7}$ & 0.014 & 0.0520 & 0.0295 & 0.0404 & $6.83 \times 10^{52}$ \\
\hline
\end{tabular}

Table 1: Results for every detected GW from the O1, O2, and O3 observing runs as of July 28th, 2019. Here $\Omega$ is the area of the $90 \%$ containment region of the GW and FAR is the false alarm rate. These values are taken from GWTC-1 and the online event database GraceDB [1, 2]. We also report 90\% C.L. U.L.s using the best-fit value from the $\chi^{2}$ fit described in Figure 2, as well as the minimum and maximum U.L.s assuming a point source hypothesis within the $90 \%$ containment region of the GW skymap. $E_{\text {iso }}$ is the U.L. on the isotropic equivalent energy emitted in neutrinos during $1000 \mathrm{~s} . \mathrm{BBH}=$ Binary Black Hole, BNS $=$ Binary Neutron Star, Ter $=$ Terrestrial. 


\section{Conclusion}

We searched for neutrino emission within $\pm 500 \mathrm{~s}$ of all reported $\mathrm{GW}$ events from the $\mathrm{O} 1, \mathrm{O} 2$, and $\mathrm{O} 3$ observing runs as of July 28th, 2019. No significant correlations with neutrino events were found and, for each GW event, we placed $90 \%$ C.L. U.L.s on the time-integrated neutrino flux and 90\% C.L. U.L.s on $E_{\text {iso. }}$ In addition to performing the neutrino follow up, we have reported our results through GCN circulars for other observatories. An independent search for joint GW+neutrino events which evaluates a Bayesian odds ratio of a detected signal is presented in [15]. The Bayesian approach produces results similar to those presented in this work. Results from both analyses are sent in IceCube's GCN circulars. Generally these results are sent out within an hour of the initial GCN notice sent by LVC. In the future we plan to send rapid GCN notices to inform the community of any significant neutrinos that should be followed up by electromagnetic observatories.

During the current $\mathrm{O} 3$ observing run, 22 binary mergers have been detected thus far. The estimated rates before the start of the $\mathrm{O} 3$ run were $\sim 1$ /week for $\mathrm{BBH}$ mergers and $\sim 1$ /month for BNS mergers. So far the observed rates have been consistent with these expectations. Assuming this rate remains stable, we should expect to see roughly 40 more GW events by the end of the $\mathrm{O} 3$ run. With an increasing sample of GWs, we plan on performing population analyses on BBH mergers and BNS mergers to examine the individual source classes as potential neutrino emitters. In future work, we also plan to extend this analysis by searching for neutrino emission on timescales longer than $\pm 500 \mathrm{~s}$. For BNS mergers especially, searches over longer timescales will have a higher chance of finding neutrino correlations if significant neutrino production occurs during the kilonova phase following the BNS merger [16, 17].
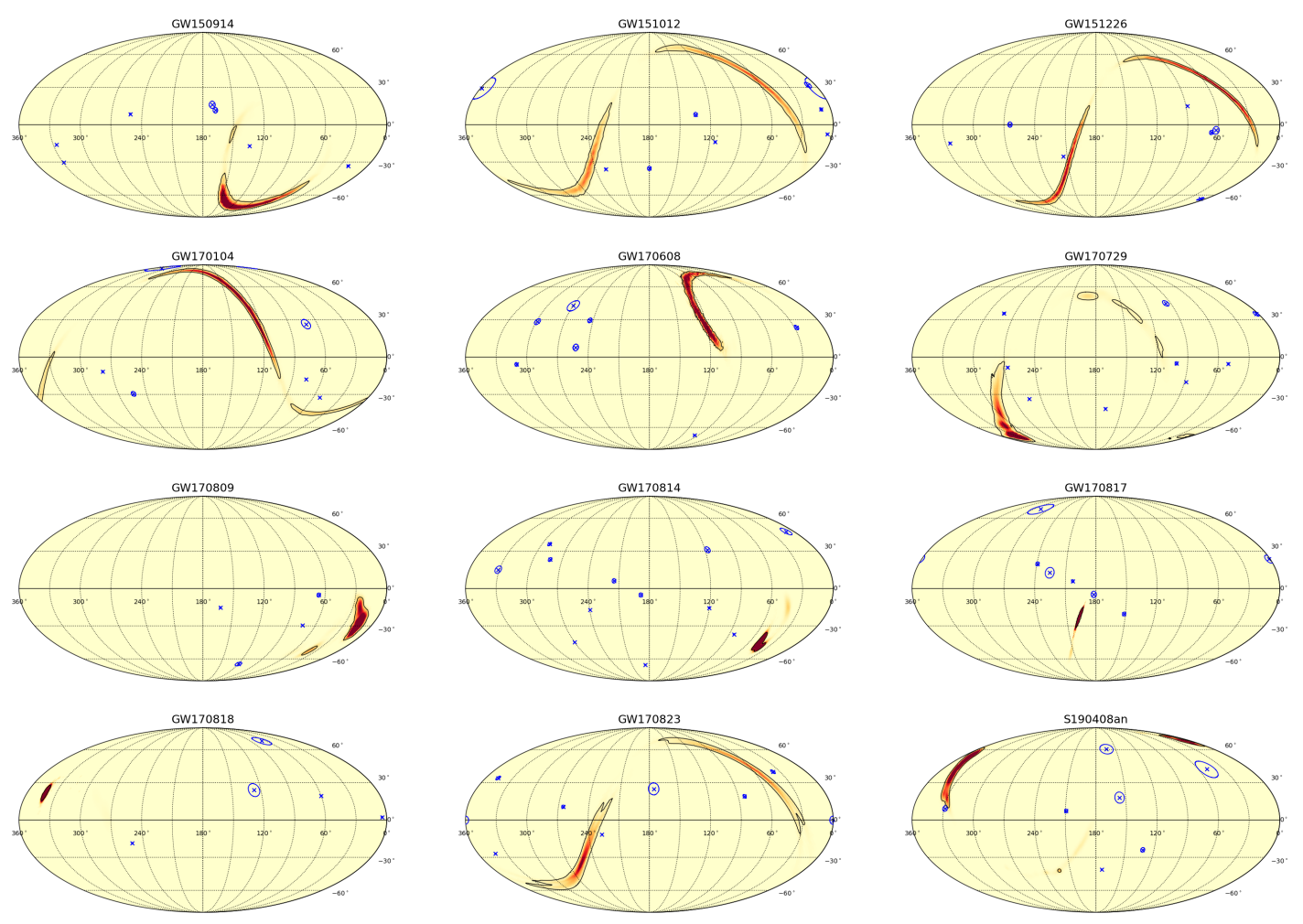

IceCube Preliminary 

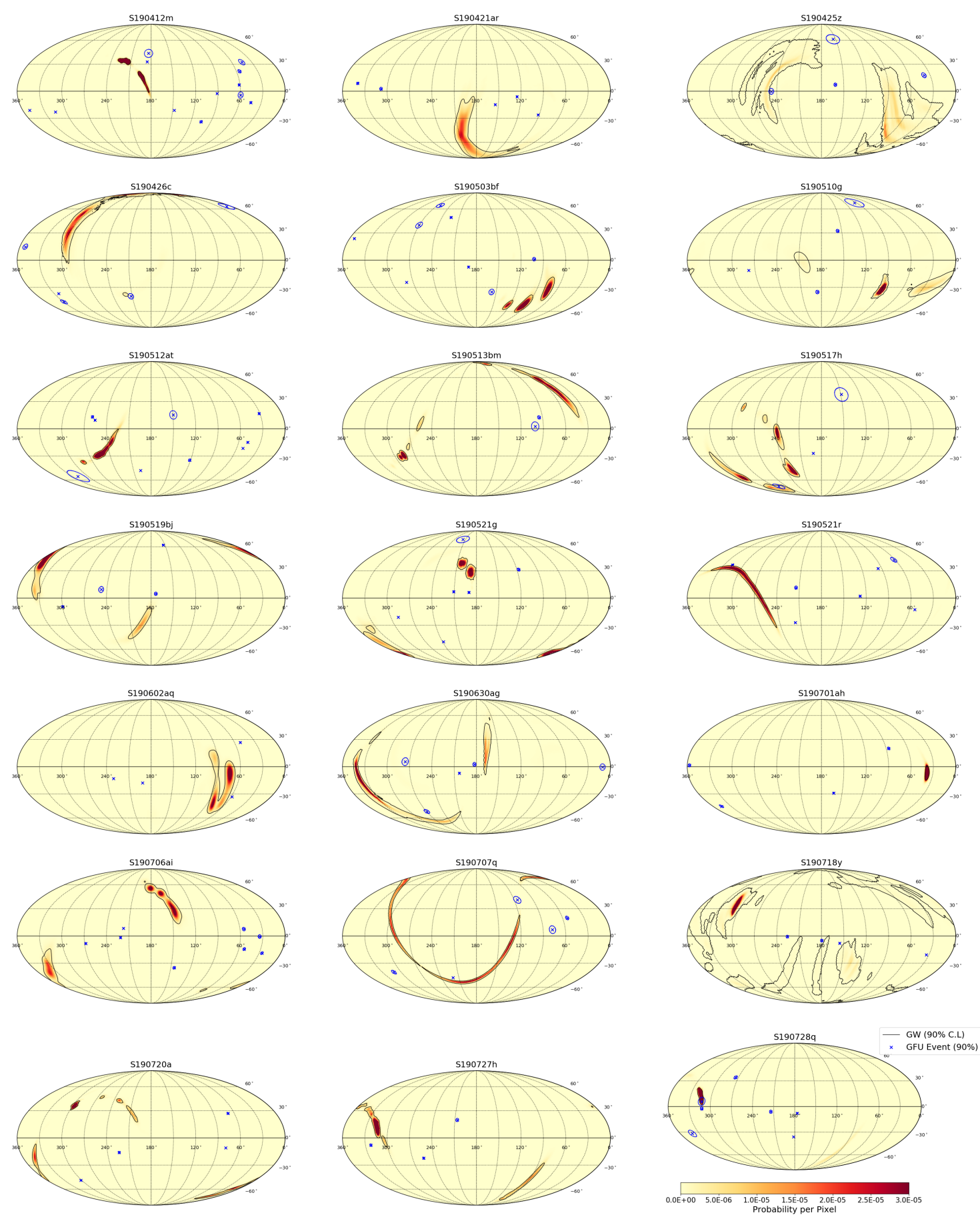

\section{IceCube Preliminary}

Figure 3: Joint skymaps for the first 33 detected GW events. Overlayed are the neutrinos within 1000 seconds of the GW trigger time. Gray contours around the blue crosses are $90 \%$ containment angular errors for the neutrinos and the black contours are $90 \%$ containment for the GW. 


\section{References}

[1] LIGO Scientific, Virgo Collaboration, B. P. Abbott et al., arXiv:1811. 12907.

[2] “Grace DB.” https://gracedb.ligo.org/search/?query=\&query_type=S\&results_format=S. Accessed: 2019-07-21.

[3] LIGO Scientific Collaboration, B. P. Abbott et al., Astrophys. J. 848 (2017) L12.

[4] ANTARES, IceCube, Pierre Auger, LIGO Scientific, Virgo Collaboration, A. Albert et al., Astrophys. J. 850 (2017) L35.

[5] LIGO Scientific, Virgo, Fermi-GBM, INTEGRAL Collaboration, B. P. Abbott et al., Astrophys. J. 848 (2017) L13.

[6] F. Halzen and D. Hooper, Rept. Prog. Phys. 65 (2002) 1025-1078.

[7] S. Razzaque, P. Meszaros, and E. Waxman, Phys. Rev. D68 (2003) 083001.

[8] K. Murase, D. Guetta, and M. Ahlers, Phys. Rev. Lett. 116 (2016) 071101.

[9] ANTARES, IceCube, LIGO Scientific, Virgo Collaboration, S. Adrian-Martinez et al., Phys. Rev. D93 (2016) 122010.

[10] ANTARES, IceCube, LIGO, Virgo Collaboration, A. Albert et al., Astrophys. J. 870 (2019) 134.

[11] IceCube Collaboration, T. Kintscher, J. Phys. Conf. Ser. 718 (2016) 062029.

[12] K. M. Górski, E. Hivon, A. J. Banday, B. D. Wandelt, F. K. Hansen, M. Reinecke, and M. Bartelmann, 622 (Apr., 2005) 759-771.

[13] B. Baret et al., Astropart. Phys. 35 (2011) 1-7.

[14] IceCube Collaboration, M. G. Aartsen et al., Astropart. Phys. 92 (2017) 30-41.

[15] IceCube Collaboration, A. Keivani et al., POS ( ICRC2019) 930 (these proceedings).

[16] S. S. Kimura, K. Murase, I. Bartos, K. Ioka, I. S. Heng, and P. Meszaros, Phys. Rev. D98 (2018) 043020.

[17] K. Fang and B. D. Metzger, Astrophys. J. 849 (2017) 153. [Astrophys. J.849,153(2017)]. 